\title{
A avaliação do pensamento crítico: uma abordagem a estudantes de medicina
}

\section{brasileiros}

\author{
The assessment of critical thinking: an approach to Brazilian medical students \\ La evaluación del pensamiento crítico: un enfoque a los estudiantes de medicina brasileños
}

Recebido: 03/09/2021 | Revisado: 10/09/2021 | Aceito: 13/09/2021 | Publicado: 15/09/2021

\author{
Aline Lopes de Almeida \\ ORCID: https://orcid.org/0000-0003-2740-3750 \\ Universidade Municipal de São Caetano do Sul, Brasil \\ E-mail: aline.almeida@online.uscs.edu.br \\ Daniel Leite Portella \\ ORCID: https://orcid.org/0000-0002-5259-9049 \\ Universidade Municipal de São Caetano do Sul, Brasil \\ E-mail: Daniel.portella@online.uscs.edu.br
}

\begin{abstract}
Resumo
Objetivo: mensurar a capacidade de pensamento crírtico dos alunos de medicina de uma instituição brasileira a partir dos domínios do PC e no decorrer das etapas do curso. Metodologia: A amostra foi composta por 312 estudantes de medicina regularmente matriculados em uma Universidade do estado de São Paulo, Brasil. Foi proposto aos participantes do estudo a aplicação do instrumento Watson-Glaser Critical Thinking Appraisal III (WG-III) para avaliar as competências de pensamento crítico dos alunos, além do questionário sociodemográfico. Utilizou-se análise descritiva para os dados sociodemográficos e para as pontuações quanto ao pensamento crítico, além de análise comparativa entre os alunos dos diferentes anos do curso Resultados: O desempenho da avaliação do Pensamento Crítico a pontuação geral média da habilidade de pensamento crítico foi 50.64 e categorizada na esfera RED $\subset$ segue a tendência de desempenho mediano apontado pelos domínios: Reconhecer Suposições (50.61), Avaliar Argumentos (50.62) e Tirar Conclusões (50.41). A análise comparativa dos dados aponta que não houve diferença significante como padrão que demonstre o deslocamento positivo do pensamento crítico conforme entre os alunos das diferentes etapas do curso. A ANOVA aponta diferença estatística pontuais apenas de desempenho para as $2^{\circ}$ e $4^{\circ}$ etapas. Conclusão: o pensamento crítico da amostra estudada está aquém das expectativas projetadas para os alunos de medicina, o desempenho na prova mostra que as estratégias pedagógicas da carreira médica e as perspectivas acadêmicas dos alunos não parecem ter um papel determinante na aquisição ou aprimoramento de pensamento crítico.
\end{abstract} Palavras-chave: Pensamento crítico; Ensino; Medicina.

\begin{abstract}
Objective: to measure the critical thinking capacity of medical students at a Brazilian institution from the PC domains and throughout the stages of the course. Methodology: The sample consisted of 312 medical students regularly enrolled at a University in the state of São Paulo, Brazil. The study participants were asked to apply the WatsonGlaser Critical Thinking Appraisal III (WG-III) instrument to assess the students' critical thinking skills, in addition to the sociodemographic questionnaire. Descriptive analysis was used for sociodemographic data and for critical thinking scores, in addition to comparative analysis between students from different years of the course. and categorized in the RED $\square$ sphere, follows the trend of average performance indicated by the domains: Recognize Assumptions (50.61), Evaluate Arguments (50.62) and Draw Conclusions (50.41). The comparative analysis of the data points out that there was no significant difference as a pattern that demonstrates the positive displacement of critical thinking according to students from different stages of the course. ANOVA points out only punctual statistical differences in performance for the 2nd and 4th stages. Conclusion: the critical thinking of the sample studied is below the expectations projected for medical students, the performance in the test shows that the pedagogical strategies of the medical career and the academic perspectives of the students do not seem to have a determining role in the acquisition or improvement of critical thinking.
\end{abstract}

Keywords: Critical thinking, Teaching, Medicine.

\section{Resumen}

Objetivo: medir la capacidad del Pensamiento Crítico de los estudiantes de medicina de una institución brasileña desde los dominios del Pensamiento Crítico y en todas las etapas del curso. Metodología: La muestra se he formada por 312 estudiantes de medicina matriculados regularmente en una Universidad del estado de São Paulo, Brasil. Se solicitó a los participantes del estudio a contestar el instrumento Watson-Glaser Critical Thinking Appraisal III (WGIII) para evaluar las habilidades de pensamento crítico, además del cuestionario sociodemográfico. Se utilizó análisis 
descriptivo para datos sociodemográficos y para puntajes de Pensamiento Crítico, además del análisis comparativo entre estudiantes de diferentes años del curso. Resultados: Los hallazgos categorizados en la esfera RED del WG-III, sigue la tendencia de desempeño promedio del puntaje indicado por los dominios: Reconocer Supuestos (50.61), Evaluar argumentos (50.62) y sacar conclusiones (50.41). El análisis comparativo de los datos señala que no hubo diferencia significativa como patrón que demuestre el desplazamiento positivo del Pensamiento Crítico según alumnos de diferentes años del curso. ANOVA señala solo diferencias estadísticas puntuales en el desempeño para la 2do y 4to año. Conclusión: el Pensamiento Crítico de la muestra estudiada está por debajo de las expectativas proyectadas para los estudiantes de medicina, el desempeño en la prueba muestra que las estrategias pedagógicas de la carrera de medicina y las perspectivas acadêmicas de los estudiantes no parecen tener un rol determinante en la adquisición o mejora del pensamiento crítico.

Palabras clave: Pensamiento crítico; Enseñanza; Medicina.

\section{Introduçãa}

As diretrizes brasileiras e internacionais de educação universitária apontam que são necessárias mudanças de paradigma no processo de ensino, aprendizagem e avaliação deslocando-se da formação centrada na transmissão de conhecimentos e memorização para um modelo fundamentado na formação por competências (Lie et al., 2011).

As Diretrizes Curriculares Nacionais do Curso de Graduação em Medicina apontam para a necessidade de formação de um médico generalista, humanista, crítico e reflexivo, capacitado para atuar, pautado em princípios éticos, no processo de saúde-doença em seus diferentes níveis de atenção, com ações de promoção, prevenção, recuperação e reabilitação à saúde, na perspectiva da integralidade da assistência. (DCN, 2014).

As DCN's afirmam no seu antelóquio que a formação do egresso deve ser crítica e reflexiva. O termo crítico, etimologicamente, provém do grego kritiké, que significa a arte de discernir, separar, julgar. A palavra reflexão vem do latim reflectere, que quer dizer "voltar atrás"; ou seja, refletir significa rever, retomar, repensar, reexaminar de maneira crítica o que já foi feito. Neste sentido, podemos dizer, de modo introdutório, que o Pensamento Crítico (PC), derivado do seu sentido etimológico, é, fundamentalmente, uma tomada consciente de decisões.

Como disciplina social e humanística, a medicina ocupa um espaço em que os diferentes fenômenos a ela inerentes exigem de seus profissionais ações que implicam em promover, prevenir, diagnosticar, intervir e avaliar o processo saúdedoença. O médico é um profissional que atua em um contexto definido pela complexidade e pela imprevisibilidade, sobretudo em um país padecido pela brutal desigualdade social como o Brasil. Os cenários de atenção são os mais distintos possíveis no que se refere ao perfil dos pacientes e familiares, dos membros da equipe multidisciplinar, assim como as condições referentes a recursos físicos e materiais exigindo do médico habilidades de pensamento crítico/reflexivo para tomar decisões complexas.

Buscando algum nível de convergência das diferentes definições disponíveis na literatura, pensamento crítico envolve competências ou habilidades cognitivas e disposições do pensador crítico. Estas disposições, que podem ser vistas como atitudes ou hábitos intelectuais, incluem ter a mente aberta, curiosidade, flexibilidade, uma propensão a buscar razão, o desejo de ser bem-informado e um respeito e vontade de entreter diversificados pontos de vista, imparcialidade, persistência e prudência (Facione, 1990).

O PC torna-se competência crucial para a formação médica porque infere a capacidade de olhar para uma situação e compreendê-la totalmente e claramente sob várias perspectivas, enquanto separa fatos de opiniões e suposições; ajudando assim no raciocínio clínico e na tomada de decisões corretas e racionais promovendo a segurança do paciente (Croskerry, 2012).

A necessidade da abordagem do pensamento crítico no âmbito acadêmico, além da referência epistemológica do cognitivismo, se faz necessária como uma resposta as proposições das DCN's. O PC pode ser considerado como conceitos teóricos de nível macro e a educação crítica, ancorada em pensadores como Paulo Freire, Henry Giroux, Peter McLaren, 
Michael Apple e Douglass Kellner, etc, como preceitos pedagógicos de nível micro (Alipour, 2013). Nesse tipo de educação, sabedoria, crítica e interpretação são consideradas objetivos educacionais valiosos. Estudantes que desenvolvem as habilidades de pensamento crítico durante a universidade retribuem para a sociedade através de benefícios sociais gigantescos (Kasalei et al., 2020).

Apesar dos flagrantes benefícios, Yared, Melo e Vieira (2020) em um recente estudo destacaram barreiras a implementação da abordagem do pensamento crítico nas universidades, como a falta de conhecimento sobre o assunto por parte do corpo docente, uso de métodos de ensino e avaliação que não facilitam o pensamento crítico dos alunos, atitudes negativas dos membros do corpo docente em relação à mudança e sua resistência a mudança, processo de seleção inadequado e formação educacional deficiente que não facilitam o pensamento crítico dos alunos, socialização insuficiente e aspectos culturais gerais.

Em geral, os aspectos culturais e o antigo sistema educacional tradicional centrado na aprendizagem passiva, eram duas barreiras significativas à implementação do programa de pensamento crítico, que parece superar essas barreiras para fornecer conceitos e habilidades de pensamento crítico nos currículos dos estudantes de medicina. Portanto, pode-se concluir que a reforma das DCN's é uma das estratégias para promover o pensamento crítico.

Existe uma variedade grande de ferramentas para avaliar o pensamento crítico. Ku (2009) aponta a diversidade de instrumentos construídos para a avaliação do pensamento crítico, fazendo referência a California Critical Thinking Skills Test (Facione, 1990), a Cornell Critical Thinking Test (Ennis, Millman, \& Tomko, 1985), a Ennis-Weir Critical Thinking Essay Test (Ennis \&Weir, 1985), Halpern Critical Thinking Assessment (HCTA, Halpern, 2010), ou ainda, a Watson-Glaser Critical Thinking Appraisal (Watson \& Glaser, 2005). Essa variedade de instrumentos cria uma ampla gama de possibilidades para avaliação cognitiva. No entanto, os testes têm limitações. Alguns, devido a faixa etária, traduções, outros ao seu formato de aplicação (múltipla escolha). Acreditar que o pensamento crítico é algo singular e universal e que possa ser mensurado apenas via linguagem verbal é uma ilusão e, certamente, deveria haver muitas outras formas de aferir esse tipo de competência, existem vários obstáculos a serem superados nesse sentido. A busca por um modelo eficiente de medição do pensamento crítico nos tempos atuais deve passar por outros meios de linguagem para garantirem eficácia estatístico-científica.

Recentemente o Watson-Glaser Critical Thinking Appraisal III (WG-III) inovou ao utilizar formato de banco de itens que permite a triagem não supervisionada de candidatos aumentando a segurança e validade do teste bem como um banco de questões adequado para uso internacional o que facilita a contextualização para traduções e uso global.

Considerando que o PC é uma competência central e transversal na educação médica, que favorece a emancipação intelectual, este estudo tem como objetivo mensurar a capacidade de PC dos alunos de medicina de uma instituição brasileira tanto partir dos domínios do PC como também no decorrer das etapas do curso.

\section{Metodologia}

\section{Amostra e Tipo de Estudo}

O estudo transcorreu a partir do método conceitual-analítico, visto que foram utilizados conceitos e ideias de outros autores, semelhantes com os objetivos deste estudo para a criação de uma análise científica.

Para buscar resultados confiáveis e respostas válidas acerca da problematização apresentada, a amostra foi elaborada de forma sequencial e por conveniência, composta por 312 indivíduos, de um universo total de 722 indivíduos, todos estudantes regularmente matriculados no curso de medicina da Universidade Municipal de São Caetano do Sul (USCS). O estudo teve uma abordagem quantitativa, de natureza aplicada, com objetivos descritivo/explicativo e de procedimentos de coleta em campo. 


\section{Local do Estudo}

A pesquisa foi realizada na Universidade Municipal de São Caetano do Sul - USCS (Campus Centro), na região do Grande ABC - SP, no período de agosto a novembro de 2020. Local de atuação profissional da pesquisadora no momento do estudo.

A USCS se destaca por "gerar, sistematizar e socializar o conhecimento e o saber em todas as suas formas, tornandoos acessíveis à sociedade e colaborando, sobretudo, para o desenvolvimento integrado da região do Grande ABC." (USCS, 2020). O curso de medicina na USCS surgiu no contexto do Programa "Mais Médicos", do governo federal, que, dentre vários objetivos específicos, tinha como geral à melhoria do atendimento aos usuários do Sistema Único de Saúde (SUS) - uma das grandes conquistas sociais da recente e frágil democracia brasileira (Constituição de 88). E, nesse contexto, formar médicos vinculados à realidade brasileira e preparados para enfrentar os principais problemas da população. Em se tratando de uma instituição municipal, com independência financeira e administrativa, não tem compromisso com o lucro de acionistas e/ou proprietários, e sim com a qualidade dos serviços que oferecem. A educação não é tratada como um produto, destoando do atual cenário de excessiva comercialização do ensino médico no país. Possui infraestrutura completa com modernos laboratórios creditados por órgãos internacionais e corpo docente altamente titulado. (USCS, 2020)

\section{Delineamento do Estudo}

O estudo foi delineado em 2 etapas:

Etapa 1 (Coleta dos dados): Foi aplicada a versão Watson-Glaser Critical Thinking Appraisal III para a verificação do desempenho dos discentes em relação ao PC.

Etapa 2 (Aplicação do questionário sociodemográfico): o questionário sociodemográfico foi aplicado com os alunos do curso de medicina da USCS em versão online com link disponibilizado em ambiente Virtual de Aprendizagem (Google Classroom) após anuência da direção do curso de terem sido submetidos a Plataforma Brasil e aprovação do CEP, e sob TCLE.

\section{Técnicas e Procedimentos}

Foi proposto aos participantes do estudo a aplicação do instrumento Watson-Glaser Critical Thinking Appraisal III (WG-III) para avaliar as competências de pensamento crítico dos alunos, além do questionário sociodemográfico.

\section{Watson-Glaser Critical Thinking Appraisal III (WG-III)}

O WG-III é um dos testes globais de avaliação do pensamento crítico mais respeitados e bem estabelecidos. Foi publicado pela primeira vez em 1964 por Goodwin Watson e Edward M. Glaser, após um período de desenvolvimento que remonta a 1926. Desde então, tornou-se o principal método de avaliação pensamento crítico global (Davies, 2019). O teste foi projetado para ser uma medida rápida, consistente e precisa da capacidade de: analisar, raciocinar, interpretar e tirar conclusões

lógicas. É adequado para avaliar adultos (16 anos ou mais) e foi revisado e aprimorado várias vezes desde seu lançamento, há várias décadas (Pashayan, 2016). Em sua versão mais recente Watson-Glaser Critical Thinking III (WG-III) acrescentou novos recursos: $\mathrm{O}$ formato de banco de itens permitindo a triagem não supervisionada aumentado a segurança e a validade do teste; perguntas selecionadas aleatoriamente, tornando improvável que dois indivíduos recebam o mesmo teste; versão cronometrada promove uma experiência consistente do candidato; relatório de perfil aprimorado com descrição mais detalhada do desempenho do sub-teste; novo banco de questões relevantes e globais, adequado para uso internacional; as pontuações estão disponíveis em um relatório de perfil mais completo que possibilita criar um plano de desenvolvimento.

A estrutura da avaliação e apresentação dos resultados do WG-III é apresentada pelo modelo RED $\odot$, que quebra o pensamento crítico na capacidade de reconhecer suposições, avaliar argumentos e tirar conclusões. 
Reconhecer suposições: é aparentemente fácil ouvir um comentário ou apresentação e assumir que as informações apresentadas são verdadeiras, mesmo sem evidências para apoiá-lo. Perceber e questionar suposições ajuda a revelar lacunas de informação ou lógica infundada. Também estimula que é preciso examinar suposições sob diferentes pontos de vista.

Avaliar os argumentos: A arte de avaliar argumentos envolve a análise de informações de maneira objetiva e precisa, questionando a qualidade do suporte de evidências e compreensão de como a emoção influencia cada situação. Barreiras comuns incluem viés de confirmação ou permissão de emoções para atrapalhar a avaliação objetiva.

Tirar conclusões: Pessoas que chegam a conclusões que seguem logica da gama de evidências disponíveis são frequentemente caracterizadas como tendo "bom senso". Elas têm o cuidado de não generalizar inadequadamente além da evidência e elas podem mudar sua posição quando a evidência exigir.

O teste é feito de maneira online e os candidatos têm no máximo 40 minutos para conclui-lo. É composto de 40 itens com base em um banco de perguntas com cenários neutros e controversos, relevantes e adequados para uso internacional; 12 testando sua capacidade de reconhecer suposições, 12 testando sua capacidade em avaliar argumentos, e 16 testando sua capacidade para tirar conclusões. Além de uma pontuação geral apresenta correlações entre ocasiões de teste - evidência de que os resultados são consistentes ao longo do tempo $(0,73-0,89)$; consistência dos itens de teste - evidência que todos os itens no teste medem a mesma coisa da mesma forma $(0,83)$; correlações entre diferentes formas do teste - evidência de que diferentes seleções de itens resultam para pontuações semelhantes $(0,82-0,88)$.

\section{Análise dos Dados}

Foi realizada uma análise descritiva para caracterização da amostra. Para os resultados dos estudantes frente ao instrumento realizou-se análise descritiva com as mesmas variáveis supracitadas. Para a análise comparativa foi utilizado o teste de normalidade de Kolgomorvo-Smirnov e após a verificação da normalidade da amostra utilizou-se a ANOVA para comparar os grupos de alunos das diferentes etapas do curso, assim como os domínios do PC. O pacote estatístico utilizado foi o SPSS 20.0 IBM.

\section{Resultados e Discussão}

Responderam aos questionários propostos 312 alunos regularmente matriculados sendo que na primeira etapa $n=29$, na segunda etapa $n=86$, na terceira etapa $n=56$, na quarta etapa $n=87$, na quinta etapa $n=33$ e na sexta etapa $n=21$. Na figura 1 são apresentadas as principais características sociodemográficas da amostra, expressas pelas frequências relativas. 
Research, Society and Development, v. 10, n. 12, e122101220203, 2021

(CC BY 4.0) | ISSN 2525-3409 | DOI: http://dx.doi.org/10.33448/rsd-v10i12.20203

Figura 1: Valores percentuais das variáveis sociodemográficas da amostra.

\begin{tabular}{|c|c|}
\hline $\begin{aligned} & 3,20 \text { Idade } \\
= & 16 \text { a } 20 \operatorname{anos}=21 \text { a } 25 \text { anos } \\
= & 26 \text { a } 30 \text { anos }=31 \text { a } 35 \text { anos } \\
= & \text { Mais de } 35 \text { anos }\end{aligned}$ & - $1^{\circ}=2^{\circ}=3^{\circ}=4^{\circ}=5^{\circ}=6^{\circ}$ \\
\hline $\begin{array}{l}\text { Cor da pele } 6,10 \\
\text { - Parda } \text { = Branca }=\text { Indigena } \\
\text { - Amarela } \text { " Preta }\end{array}$ & - Feminino = Masculino \\
\hline - Sim - Não & $\begin{array}{l}\text { Religião } \\
\text { - Católica }=\text { Evangélica } \text { - Judaica } \\
\text { = Islâmica } \quad \text { - Espirita = Outros }\end{array}$ \\
\hline $\begin{array}{l}\text { Ensino básico } 3,00 \text { 3,50 } \\
\text { - Totalmente em escola pública } \\
\text { - Maior parte em escola pública } \\
\text { - Maior parte em escola privada } \\
\text { - Totalmente em escola privada }\end{array}$ & $\begin{array}{l}\text { Estado Civil } \\
\qquad 4,20\end{array}$ \\
\hline
\end{tabular}


Research, Society and Development, v. 10, n. 12, e122101220203, 2021

(CC BY 4.0) | ISSN 2525-3409 | DOI: http://dx.doi.org/10.33448/rsd-v10i12.20203

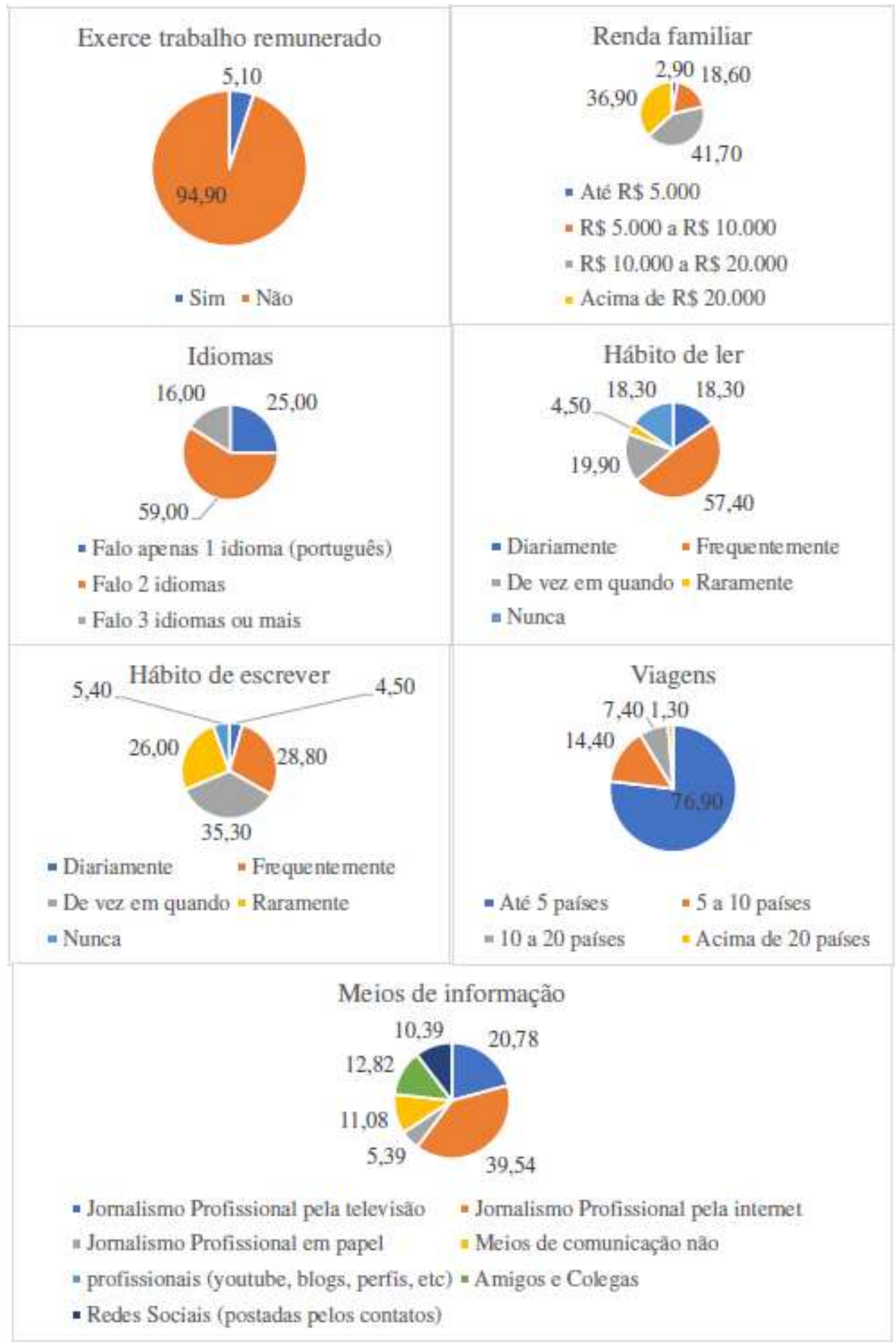

Fonte: Autores. 
Trata-se de uma população que em sua maioria fala dois idiomas ou mais $(\mathrm{n}=234 ; 75 \%)$ tem o hábito de ler frequentemente $(\mathrm{n}=179 ; 57.4)$ mas que escreve apenas as vezes ou raramente $(\mathrm{n}=197 ; 61.3 \%)$. A acachapante maioria não trabalha $(n=296 ; 94.9 \%)$ e não concluiu nenhum outro curso superior $(n=288 ; 98.3 \%)$. No que tange a fonte de informação, a vasta maioria utiliza meios digitais (61.01\%) sendo estes, meios profissionais e formais (63.8\%), entretanto, uma parte considerável da amostra utiliza tanto meios formais quanto informais para se informar (35.6\%). Apesar de não ser o objetivo principal deste trabalho e não ter sido encontrada significância estatísticas para os outros dados sociodemográficos, cabe salientar que estas características, de certa forma, são fundamentais para os explicar/discutir os resultados que se seguem.

A pontuação geral média da habilidade de pensamento crítico foi 50.64 e categorizada na esfera RED $@$ segue a tendência de desempenho mediano apontado pelos domínios: Reconhecer Suposições (50.61), Avaliar Argumentos (50.62) e Tirar Conclusões (50.41) (Tabela 1).

Tabela 1: Desempenho do pensamento crítico em geral e por domínios através dos valores obtidos na aplicação do WGIII.

\begin{tabular}{|c|c|c|c|c|}
\hline & Média (pontos) & Desvio Padrão & $\begin{array}{l}\text { Valores Mínimos } \\
\text { (pontos) }\end{array}$ & $\begin{array}{c}\text { Valores Máximos } \\
\text { (pontos) }\end{array}$ \\
\hline Média Geral WG3 & 50,65 & $\pm 6,83$ & 35,00 & 69,00 \\
\hline Competência de "Reconhecer Suposições" & 50,61 & $\pm 7,44$ & 32,00 & 67,00 \\
\hline Competência de "Avaliar os argumentos" & 50,63 & $\pm 6,18$ & 34,00 & 69,00 \\
\hline Competência de "Tirar Conclusões" & 50,42 & $\pm 6,72$ & 32,00 & 70,00 \\
\hline
\end{tabular}

Fonte: Autores.

A Análise comparativa dos dados aponta que não houve diferença significante relevante como padrão que demonstre variação do desempenho de Pensamento Crítico conforme entre os alunos das diferentes etapas do curso. A ANOVA aponta diferença estatística pontuais apenas de desempenho para as $2^{\circ}$ e $4^{\circ}$ etapas (Tabela 2).

Tabela 2: Análise do desempenho no instrumento sobre Pensamento Crítico dos discente em cada etapa do curso.

\begin{tabular}{|c|c|c|c|c|c|c|}
\hline $\begin{array}{c}\text { Domínio do Pensamento } \\
\text { Crítico }\end{array}$ & Etapas do curso & $\mathrm{N}$ & Média (pontos) & $\begin{array}{l}\text { Desvio Padrão } \\
( \pm)\end{array}$ & $\begin{array}{l}\text { Valor Mínimo } \\
\text { (pontos) }\end{array}$ & $\begin{array}{l}\text { Valor Máximo } \\
\text { (pontos) }\end{array}$ \\
\hline \multirow{6}{*}{ Média Geral } & 1,00 & 25 & 48,5200 & 5,16495 & 43,00 & 61,00 \\
\hline & 2,00 & 70 & $50,3429 *$ & 7,55633 & 35,00 & 69,00 \\
\hline & 3,00 & 52 & 49,6538 & 6,38283 & 37,00 & 66,00 \\
\hline & 4,00 & 85 & 52,3294 & 7,60387 & 37,00 & 69,00 \\
\hline & 5,00 & 31 & 51,1613 & 5,42277 & 43,00 & 66,00 \\
\hline & 6,00 & 20 & 49,0000 & 3,97360 & 43,00 & 58,00 \\
\hline \multirow{2}{*}{ R - Reconhecer as suposições } & 1,00 & 25 & 49,7600 & 7,42339 & 35,00 & 63,00 \\
\hline & 2,00 & 70 & 49,7714 & 7,50685 & 32,00 & 66,00 \\
\hline
\end{tabular}




\begin{tabular}{|c|c|c|c|c|c|c|}
\hline & 3,00 & 52 & 49,9808 & 7,50553 & 36,00 & 67,00 \\
\hline & 4,00 & 85 & 51,7765 & 7,55043 & 36,00 & 63,00 \\
\hline & 5,00 & 31 & 50,9677 & 8,27641 & 32,00 & 63,00 \\
\hline & 6,00 & 20 & 50,7500 & 5,13886 & 42,00 & 63,00 \\
\hline \multirow{6}{*}{ E - Avaliar os argumentos } & 1,00 & 25 & 48,6400 & 4,63573 & 41,00 & 57,00 \\
\hline & 2,00 & 70 & 51,0714 & 6,67533 & 34,00 & 69,00 \\
\hline & 3,00 & 52 & 50,0769 & 6,06128 & 34,00 & 64,00 \\
\hline & 4,00 & 85 & 51,2471 & 6,69205 & 34,00 & 64,00 \\
\hline & 5,00 & 31 & 51,0968 & 5,59973 & 41,00 & 64,00 \\
\hline & 6,00 & 20 & 49,6500 & 4,72702 & 41,00 & 59,00 \\
\hline \multirow{6}{*}{ D - Tirar as conclusões } & 1,00 & 25 & 48,2400 & 7,15472 & 38,00 & 68,00 \\
\hline & 2,00 & 70 & 50,0286 & 7,07101 & 32,00 & 63,00 \\
\hline & 3,00 & 52 & 49,8269 & 5,72480 & 39,00 & 64,00 \\
\hline & 4,00 & 85 & 52,1412 & 7,31462 & 35,00 & 70,00 \\
\hline & 5,00 & 31 & 51,1290 & 5,32129 & 41,00 & 63,00 \\
\hline & 6,00 & 20 & 47,6000 & 5,11345 & 38,00 & 57,00 \\
\hline
\end{tabular}

*=diferença significante $(\mathrm{p}<0,05)$ em relação ao $4^{\circ}$ ano. Fonte: Autores.

Os principais resultados do presente estudo foram: 1) valores de PC insuficientes em relação às expectativas das DCNs para estudantes de medicina; 2) a falta de aprimoramento na capacidade do PC no decorrer do curso de medicina.

\section{Desempenho no Teste de Pensamento Crítico (WG-III) Aquém das Expectativas das DCN’s (2014)}

Segundo a interpretação referenciada através dos critérios estabelecidos pelo instrumento do WG-III (escore Theta), o desempenho dos alunos de medicina da presente amostra para o Pensamento Crítico foi considerado na média populacional, portanto insuficiente. Ainda que a população normativa para o teste em sua última versão e na língua portuguesa ainda não esteja concluída e que interpretações e comparações devam ser cautelosas, os resultados foram semelhantes a estudos que medem o PC em estudantes universitários na área de saúde (Behrens, 1996; Frye, 1999; Del Bueno, 2005; Kong et al., 2014).

Partindo desse pressuposto cabem entendimentos para hipóteses explicativas. Tais hipóteses abordam o modelo de avaliação e as expectativas de tal competência. A literatura apresenta uma variedade de instrumentos de avaliação sobre o PC. Riegel e Crossetti (2018) apontam m uma revisão integrativa oito diferentes instrumentos para avaliar o PC. É sabido que tais comparações de resultados entre distintos instrumentos não é possível por inúmeras razões: diferentes proposições de origem, criação a partir de populações distintas, modelos de construção do instrumento, além de questões regionais e culturais dos locais nos quais os instrumentos foram idealizados e construídos. Dessa forma é necessário cautela para avaliar uma habilidade cognitiva através de instrumentos avaliativos.

Entretanto, o instrumento aqui apresentado segue tais critérios supracitados para que haja uma interpretação adequada dos resultados. quando se percebe que alguns resultados apresentados na literatura são provenientes de instrumentos aplicados de forma adequada para a população proposta.

Dessa forma, seguindo com a interpretação dos dados percebe-se que estudantes de medicina em geral apresentam desempenho mediano acerca do PC. De acordo com as DCNs, do curso de medicina o ensino deve ser reflexivo, crítico e criativo. Sendo assim, o PC deve ser estimulado e a expectativa de um desempenho acima da média é o cenário projetado para estudantes de medicina. A causa desses resultados é obviamente multifatorial, contudo, deve-se discutir em especial sobre o papel do discente e do docente nesse cenário. 
Pode parecer antilógico que uma população privilegiada do ponto de vista financeiro e que teve acesso as melhores escolas sem ter tido grandes privações, atingir um desempenho aquém das expectativas no teste de pensamento crítico, mas a dominação ideológica é fundamental para encobrir o caráter contraditório do sistema político econômico atual e barrar a emancipação intelectual do indivíduo, ainda que na camada mais privilegiada da população brasileira. Sobretudo desta geração que, via de regra, não tem tempo de pensar, apenas de consumir. O pensamento crítico é o que nos permite distinguir fontes confiáveis de desinformação e nos permite formar nossas próprias opiniões com base nas coisas que vemos, lemos e sentimos.

É o que nos permite tomar e justificar decisões boas, conscientes e totalmente informadas. É a habilidade essencial para a era da informação.

\section{Comparação entre os alunos das diferentes etapas}

Ao observar o desempenho dos discentes através das etapas de ensino no curso de medicina não se encontrou um comportamento incremental conforme os discentes avançam de etapa em relação ao PC. Apenas uma diferença significante pontual e casual ocorreu entre os resultados dos alunos do $2^{\circ}$ e $4^{\circ}$ anos.

O desempenho no teste mostra que a universidade parece não ter um papel decisivo na aquisição ou aprimoramento do pensamento crítico. Esse resultado era esperado e mostra consonância com duas metanálises realizadas por Fong (2017) e ROSS (2013) que associam as habilidades de pensamento crítico ao desempenho acadêmico e ao papel nulo ou discreto que a universidade parece desempenhar. Os alunos que recebem instrução de pensamento crítico demonstraram estar mais dispostos a aceitar teorias com base científica (Rowe et al., 2015), e estudos mostraram que a capacidade de pensamento crítico prediz a média de notas da faculdade (ACT, sem data).

Apesar desses achados na literatura e no estudo em questão é necessário refletir sobre outros fatores que são intervenientes no processo de desenvolvimento do PC, como o papel do discente e do docente. Ambos apresentam intersecções na questão do PC. Como exemplo o estudo de Kim (2019) mostra estratégias distintas para o desenvolvimento do PC em alunos de medicina coreanos. Para avaliar o PC de seus alunos Kim utilizou-se de estratégias com disparadores textuais e não textuais. Os alunos apontaram melhor percepção e desempenho à estratégia não textual. Isso aponta que o docente deve buscar novas formas de apresentação das tarefas para todas as competências, inclusive para o PC. Nas escolas que utilizam ferramentas de metodologias ativas parte-se do pressuposto que os docentes estão dispostos e aptos a essas práticas que induzam os discentes à reflexão e criticidade. Entretanto, existem inúmeros desafios para a prática docente, nem todos estão devidamente capacitados e isso exerce influência relevante no aprendizado significativo dos aprendizes.

Da mesma forma, o discente deve estar aberto e proativo para que busquem o conhecimento em diversas fontes e formas. Nesse caso o que se têm observado é uma diminuição dessas características tão importante para o desenvolvimento do PC. Zia e Dar (2019) apresentaram em seu estudo como era a percepção dos estudantes paquistaneses de medicina sobre o PC. Os discentes apontaram uma alta percepção da importância de desenvolver o PC. Contudo, nesse mesmo estudo os autores também observaram a disposição dos estudantes em desenvolver o PC e nesse quesito, notou-se que não há grande disposição que isso ocorra.

Cabe ainda uma observação quanto ao cenário de coletas dos dados para o presente estudo: a Pandemia por Covid19. Outrora abordado, o papel dos docentes no desenvolvimento do PC em seus alunos apresenta uma dificuldade natural do desafio por conta do binômio docente-discente e agravou-se no cenário de isolamento social. Pensando nas barreiras para desenvolvimento do PC Riegel et al (2021) discutiram a necessidade da inovação frente aos problemas de distanciamento nas aulas regulares e a mudança abrupta do modelo clássico e tradicional de ensino. Barbosa et al (2020) apresenta relatos de professores que foram surpreendidos com a necessidade de dialogar com as Tecnologias da Informação e Comunicação (TIC) as quais não eram familiares à maioria desses docentes. $\mathrm{O}$ que foi imposto aos docentes Brasil a fora pela pandemia por 
Covid19 foi o chamado Ensino Remoto de Emergência (Hodges et al., 2020) o qual é realizado em situações emergenciais e de catástrofes e mediante a isso não há preparação adequada nem dos conteúdos ou familiarização das tecnologias por parte dos docentes, tão pouco a aderência e significado pelo lado dos discentes.

\section{Conclusão}

De acordo com o exposto no texto acima baseado nos resultados e discussões é possível concluir que o PC da amostra estudada se encontra aquém das expectativas projetadas para estudantes de medicina. Também pode-se concluir que a exposição às estratégias do curso de medicina observado no estudo não sãø foram suficientes para desenvolver o Pensamento Crítico dos discentes.

Acredita-se que o grande desafio da Universidade é mediar um ensino significativo, reflexivo, que promova a emancipação intelectual dos estudantes de medicina por meio do pensamento crítico, considerando esta a base para o raciocínio clínico e a tomada de decisões conscientes.

Para que isso possa ocorrer ressalta-se a necessidade de antecipar e infundir atividades de pensamento crítico no início dos currículos do ensino fundamental e médio, como uma forma de incentivar os alunos a desenvolver suas habilidades de pensamento ainda mais cedo. Essa prática foi mostrada por Shin (2006) de forma exitosa.

Ressalta-se que os estudantes da amostra aqui pesquisada, participavam de um modelo de ensino pautado em metodologias ativas elaborado desde a Proposta Pedagógica Curricular. Dessa forma, cabe uma reflexão sobre qual o grau de comprometimento houve com a adoção do ensino remoto de emergência nas dinâmicas dessas metodologias. Tal fato pode ter impactado em alguma medida o resultado do PC da amostra.

\section{Agradecimentos}

À Universidade Municipal de São Caetano do Sul pelo fomento à pesquisa através da bolsa de estudos para a primeira autora no Programa de Mestrado em Inovação no Ensino Superior em Saúde.

\section{Referências}

Barbosa, A. M., Viegas, M. A. S., \& Batista R. L. N. F. F. (2020). Aulas presenciais em tempos de pandemia: relatos de experiências de professores do nível superior sobre aulas remotas. Revista Ausgustus, 25(51):255-280.

Behrens, P. J. (1996). The Watson-Glaser critical thinking appraisal and academic performance of diploma school students. Journal of Nursing Education, $35(1): 34-36$.

Croskerry, P. (2012). Perspectives on diagnostic failure and patient safety. Healthc Q, 15(1):50-56.

Davies, W., \& Stevens, M. (2019). The Importance of Critical Thinking and How to Measure. Pearson TalentsLens,

Del Bueno, D. (2005). A crisis in critical thinking. Nursing education perspectives, 26(5):278-282.

Facione, P. A. (1990). Critical thinking: A statement of expert consensus for purposes of educational assessment and instruction-The Delphi report. California Academic Press.

Frye, B., Alfred, N., \& Campbell, M. (1999). Use of the Watson-Glaser critical thinking appraisal with BSN students. Nursing and Health Care Perspectives, 20(5):253-253.

Fong, C. J., Kim, Y., Davis, C. D., Hoang, T., \& Kim Y. W. (2017). A meta-analysis on critical thinking and community college student achievement. Thinking Skills and Creativity, 26:71-83.

Halpern, D. F. (1996). Thought and knowledge: An introduction to critical thinking. Lawrence Erlbaum Associates, Publishers.

Halpern, D.F. (1998). Teaching critical thinking for transfer across domains, dispositions, skills, structure training, and metacognitive monitoring, American Psychologist, 53:449-455.

Hodges, C., Trust, T., Moore, S., Bond, A., \& Lockee, B. (2020). Diferenças entre o aprendizado online e o ensino remote de emergência. Revista da Escola, Professor, Educação e Teconologia. 2:1-12. 
Research, Society and Development, v. 10, n. 12, e122101220203, 2021

(CC BY 4.0) | ISSN 2525-3409 | DOI: http://dx.doi.org/10.33448/rsd-v10i12.20203

Kasalei, A., Mitra A., Nabeiei, P., Bazrafkan L., \& Mousavinezhad H. (2020). Barriers of Critical Thinking in Medical Students' Curriculum from the Viewpoint of Medical Education Experts: A Qualitative Study. Journal of Advances in Medical Education \& Professionalism, 8(2):72-82.

Kim, D. (2019). Evaluation of critical thinking course for premedical students using literature and film. Korean Journal of Medical Education, 31(1):19-28.

Kong, L, Qin, B., Zhou Y., Mou S., \& Gao H. (2014). The effectiveness of problem-based learning on development of nursing students' critical thinking: A systematic review and meta-analysis. International journal of nursing studies, 51(3):458-469.

Ku, K. Y. (2009). Assessing students' critical thinking performance: Urging for measurements using multi-response format. Thinking Skills and Creativity, 4:70-76.

Lie, D. A., Lee-Rey, E., Gomez, A., Berekney S., \& Braddock III, H. (2011). Does cultural competency training of health professionals improve patient outcomes? A systematic review and proposed algorithm for future research. Journal of general internal medicine, 26(3):317-325.

Pashayan, N., Gray, S., Duff, C., Parkes, J., Williams, D., Patterson, F., et al (2016). Evaluation of recruitment and selection for specialty training in public health: interim results of a prospective cohort study to measure the predictive validity of the selection process. Journal of Public Health (Oxf), 38(2):e194 e200.

Rowe, M. P., Gillespie, B., Harris, K. R., Koether, S. D., Shannon, L., \& Rose, L. A. (2015). Redesigning a general education science course to promote critical thinking. CBE-Life Sciences Education, 14(3):14ar30,1-14ar30,12.

Riegel, F., \& Crossetti, M. G. O. (2018). Referenciais teóricos e instrumentos para avaliação do pensamento crítico na enfermagem e na educação. Resvista Gaúcha de Enfermagem, 39:e2017-0097.

Riegel, F., Martini, J, G., Bresolini, P., Mohallem, A. G., \& Nes A. A. G. (2021). Desenvolvendo o pensamento crítico no ensino da enfermagem: um desafio em tempos de pandemia de Covid19. Escola Anna Nery,25(spe):e20200476.

Ross, D., Loeffler, L. K., Schipper, S., Vandermeer, B., \& Allan, G. M. (2013). Do scores on three commonly used measures of critical thinking correlate with academic success of health professions trainees? A systematic review and meta-analysis. Academic Medicine, 88(5):724-734.

Shin, S., Ha, J., Shin, K., \& Davies, M. K. (2006). Critical thinking ability of associate, baccalaureate and RN-BSN senior students in Korea. Nursing Outlook, 54(6):328-333.

Yared, Y. B., De Melo, S. M. M., \& Vieira, R. M. (2020) A Importância do Pensamento Crítico em Inovações Curriculares: interface com a educação sexual emancipatória. Educação (UFSM), 45(36):1-29. 